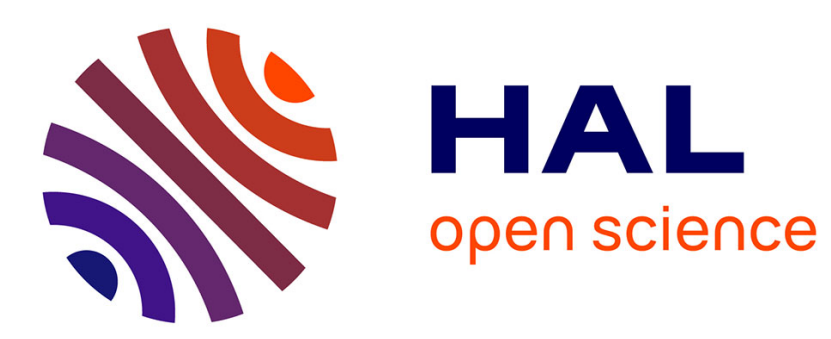

\title{
QoE-aware admission control for multimedia applications in IEEE 802.11 wireless networks
}

Kandaraj Piamrat, Adlen Ksentini, César Viho, Jean-Marie Bonnin

\section{To cite this version:}

Kandaraj Piamrat, Adlen Ksentini, César Viho, Jean-Marie Bonnin. QoE-aware admission control for multimedia applications in IEEE 802.11 wireless networks. VTC'08: IEEE 68th Vehicular Technology Conference, Sep 2008, Calgari, Canada. 10.1109/VETECF.2008.310 . hal-02901396

\section{HAL Id: hal-02901396 https://hal.science/hal-02901396}

Submitted on 17 Jul 2020

HAL is a multi-disciplinary open access archive for the deposit and dissemination of scientific research documents, whether they are published or not. The documents may come from teaching and research institutions in France or abroad, or from public or private research centers.
L'archive ouverte pluridisciplinaire HAL, est destinée au dépôt et à la diffusion de documents scientifiques de niveau recherche, publiés ou non, émanant des établissements d'enseignement et de recherche français ou étrangers, des laboratoires publics ou privés. 


\section{QoE-aware Admission Control for Multimedia Applications in IEEE 802.11 Wireless Networks}

\author{
Kandaraj Piamrat ${ }^{1,2}$ \\ ${ }^{1}$ INRIA Rennes-Bretagne Atlantique \\ Campus de Beaulieu \\ 263 Avenue du Général Leclerc \\ 35042 Rennes, France \\ Email: kpiamrat@inria.fr
}

\author{
Adlen Ksentini and César Viho ${ }^{2}$ \\ ${ }^{2}$ IRISA/Université de Rennes I \\ Campus de Beaulieu \\ 263 Avenue du Général Leclerc \\ 35042 Rennes, France \\ Email:\{aksentin,cesar.viho\}@irisa.fr
}

\author{
Jean-Marie Bonnin ${ }^{3}$ \\ ${ }^{3}$ Telecom Bretagne \\ 2, rue de la Châtaigneraie \\ CS 17607 \\ 35576 Cesson-Sévigné, France \\ Email: jm.bonnin@ @elecom-bretagne.eu
}

\begin{abstract}
Widespread use of wireless networks nowadays raises many problems for service providers in managing their resources. These problems are caused mainly by restricted bandwidth and variable radio condition in this type of network. Moreover, with the emergence of multimedia traffic and its requirements in terms of quality, admission control is hence an inevitable choice to optimize network resources while maintaining high service quality at users. In this paper, we propose an admission control mechanism based on Quality of Experience (QoE) perceived by users. The human QoE is obtained by a tool called Pseudo Subjective Quality Assessment (PSQA), which is based on statistic learning using Random Neural Network (RNN). Instead of relying on technical parameters such as bandwidth, loss, or latency, which do not correlate well with human perception, our scheme is based on Mean Opinion Score (MOS) but without interaction from real humans. The simulation results demonstrate the better performance of our proposition compared to the loss-based approach regarding user satisfaction evaluated by achieved QoE at user and bandwidth utilization of the network evaluated by goodput.
\end{abstract}

Index Terms-Wireless Network, IEEE 802.11, Admission Control, Quality of Experience, Mean Opinion Score

\section{INTRODUCTION}

Since wireless local area networks (WLAN) have started to be deployed, users can connect easily to the Internet and the number of Internet users has increased significantly. Nowadays, WLAN based on IEEE 802.11 [1] standards with infrastructure mode is the most popular as we can see hot spots everywhere. At the same time, enormous progress has been made with this technology, and the ability to support advanced services became possible. As a result, mobile hosts running real-time multimedia applications such as video streaming and voice-over IP (VoIP) are ubiquitous. These multimedia users are the major concern because their traffic is restricted in terms of quality. In addition, the nature of wireless network (limited bandwidth, shared resources, channel interference...) made it easy to be over utilized. Therefore, the load must be controlled carefully so that acceptable quality for real-time applications can be maintained while not penalizing service providers with underutilization.

To guarantee service quality at users and to optimize resource utilization, admission control is essential; otherwise degradation in quality will result from high collision. Controlling admission can be handled with several methods, we observed two main approaches: access scheduling and resource provisioning, as explained below.

- The first approach consists in scheduling access to the wireless channel. This approach has been proposed to solve inherited problems from MAC protocol in IEEE 802.11 standard that does not support neither quality of service $(\mathrm{QoS})$ nor multiple traffic categories. First, IEEE 802.11e [2] standard has been created for supporting multiple traffic categories and then many variations have been designed, most of them try to schedule access to the channel taking into account different traffic categories and prioritizing the multimedia one. Similar approach proposed by Sheu and Sheu [3] manages resources by splitting the contention period into two subperiods: one for contention between real-time stations and another for contention between non real-time stations.

- The second approach consists in restricting the volume of traffic that enters into the network with an objective of QoS provisioning. This is usually done by estimating channel utilisation based on network measurements. Some schemes have mainly focused on the analysis of throughput in saturated conditions; referring to collision probability analyzed by Bianchi [4], Pong and Moors [5] provide a mechanism to predict achievable throughput for all users after a new connection is accepted. Another scheme proposed by Zhai et al. [6] has developed an analytical model to assess the capability of 802.11 and to control admission of new flows based on channel busyness ratio.

Even many admission control mechanisms exist, most of them are aware of QoS but none takes into consideration the quality of experience, which is the most important factor in the increasing multimedia traffic such as video and voice applications today. A majority of the mechanisms rely on technical parameters, especially bandwidth. They usually compare available and requested bandwidths before deciding whether to accept a connection or not, as in resource provisioning approach. This works well with wired networks where bandwidth 
provisioning is easier than in the wireless environment. In addition, bandwidth alone is not enough to guarantee quality; consequently, other works have been proposed to evaluate quality, for example, Park et al. [7] make use of Peak Signal to Noise Ratio (PSNR), basically used to compare quality of receiving to transmitting videos, to estimate quality. However, PSNR can only be measured once the video traffic has arrived at receivers, so this approach is not appropriate for controlling admission in real-time. To accomplish both goals of enabling high quality for admitted flows and dynamic adaptation to network conditions, we propose a QoE-based admission control mechanism that administers the access network in real-time based on user's perceived quality.

The remainder of this paper is organized as follows. We first introduce the QoE concept and the PSQA tool in Section II. Then, Section III presents the proposed admission control algorithm along with interaction between access points and PSQA tool. The corresponding simulation results are presented in Section IV; they concern user satisfaction in terms of QoE achieved and bandwidth utilization in terms of goodput. Finally, Section V presents the conclusion and future work.

\section{Perceived-Quality Measurement}

In this section, we begin by introducing the notion of quality of experience (QoE), the approaches to assess this quality of experience, and then we describe the PSQA tool.

\section{A. Quality of Experience - Definition}

Quality of Experience (QoE) is the overall acceptability of an application or service, as perceived subjectively by the end-user [8]. It is basically a subjective measurement of endto-end performance at the service level, from the point of view of users. As an important measure of the end-to-end performance at the service level from the user's perspective, the QoE is an important metric for the design of systems. As such, it is also an indicator of how well the system meets its targets. This is particularly relevant for video services because bad network performance may highly affect the user's experience. Therefore, when designing systems the expected QoE is often taken into account as a system output metric. This QoE metric is often measured at the end device, however, the overall acceptability may be influenced by user expectations and context.

\section{B. Quality of Experience - Assessment}

Generally, traffics are monitored in terms of technical parameters such as throughput, delay, jitter, and loss. These parameters give technical performances of the network but they do not reveal efficiently the QoE perceived by users. In order to evaluate this QoE, several mechanisms have been proposed in both objective and subjective approaches. The former approach consists in measuring QoS by monitoring network parameters such as throughput, latency, and error whereas the latter consists in measuring QoE evaluated by real humans in terms of Mean Opinion Score (MOS). The
TABLE I

MEAN OPINION SCORE - MOS

\begin{tabular}{|c|c|c|}
\hline MOS & Quality & Impairment \\
\hline \hline 5 & Excellent & Imperceptible \\
\hline 4 & Good & Perceptible but not annoying \\
\hline 3 & Fair & Slightly annoying \\
\hline 2 & Poor & Annoying \\
\hline 1 & Bad & Very annoying \\
\hline
\end{tabular}

definition of each score is explained in terms of quality and impairment as shown in Table I.

Although the two approaches cause significant drawbacks, many researchers are based on them in order to design their systems. The problem of the objective approach is an inaccuracy of the score; because the score is based only on technical parameters, it does not correlate well with real human evaluation. On the other hand, the subjective approach is accurate but high-cost and time-consuming. This makes it hard to repeat often and it cannot be used in real time.

\section{Pseudo-Subjective Quality Assessment - PSQA}

Considering advantages and drawbacks of the previous approaches, to achieve the perceptual quality evaluation we are interested in a novel concept called Pseudo-Subjective Quality Assessment (PSQA) [9], which is hybrid between subjective and objective approaches. It keeps advantages and eliminates drawbacks of both approaches. PSQA is based on statistic learning using random neural network $(\mathrm{RNN})$. The idea is to train the RNN to learn the mapping between QoE score and technical parameters so that we can use a trained-RNN as a function to give QoE score in real-time.

In order to use this tool, three steps need to be done a priori. We summarize these steps below.

1) Configuration: we first choose configurations, which are sets of quality affecting parameters such as codec, bandwidth, loss, delay, and jitter along with their ranges of values that will be used for the RNN training. Then we take several video samples to be distorted with the configurations previously chosen.

2) Training: we ask for a panel of human observer to evaluate the distorted videos as illustrated in Fig.1 and then we put the configurations and corresponding MOSs into two databases: training and validation databases. After that, we train the RNN to learn the mapping of configurations and scores as defined in the training database. Once the tool has been trained, we have a function $f()$ that can map any possible value of parameters into MOS.

3) Validation: we validate the RNN by comparing value given by the function $f()$ at the point corresponding to each configuration in the validation database. If the values are closed enough, the RNN is validated; otherwise, we have to review chosen configurations. 
Once RNN has been validated, PSQA can be used anywhere in real-time without any interaction from real human. It gives scores in terms of MOS as if there were real humans marking their perception of the quality.

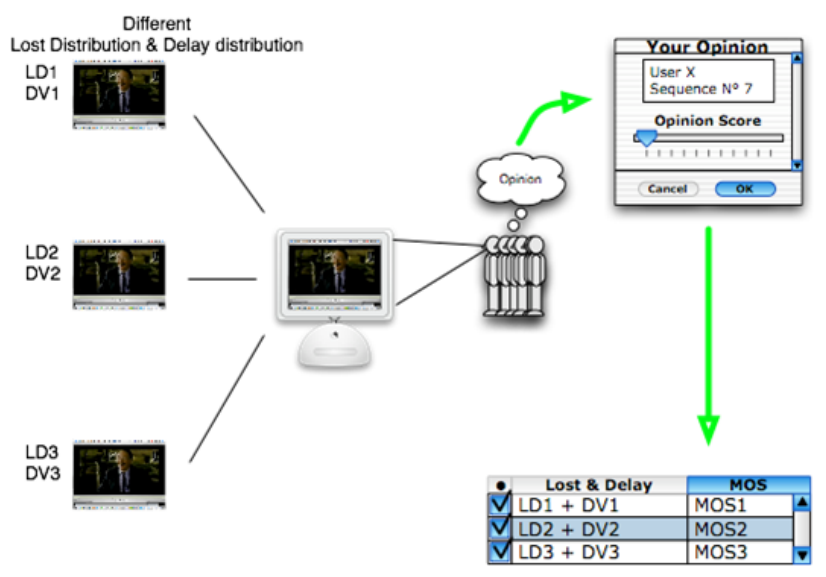

Fig. 1. Evaluation of distorted videos by real humans

\section{Admission Control Mechanism}

We propose an admission control mechanism based on PSQA tool previously described. We place our context in wireless access environments such as IEEE 802.11 standards with infrastructure mode, meaning that all traffic passes through an access point (AP). This choice has been made because we want the access point to act as controller equipped with PSQA tool. The idea is to have access points monitor MOS for each connection in order to have knowledge of the perceived quality level of the service and then decide whether to accept a new connection or not accordingly.

\section{A. Access points in our scheme}

The access point in our admission control algorithm can be illustrated with a Mealy automaton in Fig.2 in which we concentrate only on the states concerning our scheme. Assume at the beginning that access point is up and waiting for connection requests. When a new connection is requested, the access point computes an average MOS of all ongoing connection. If MOS is higher than an acceptable level plus a threshold, then a new connection will be accepted; otherwise it will be rejected. The threshold is used to absorb degradation of quality after a new connection is accepted. For economizing processing time, the access point computes MOS only when a new connection

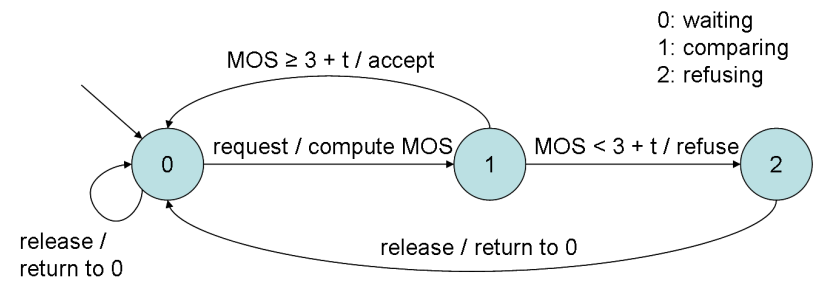

Fig. 2. States of the access point in our scheme is requested and not periodically. The dynamic and economy of our approach are suitable for wireless networks where channel conditions change often.

In our mechanism, we choose the score 3 (fair quality) to decide for admission as we assume that fair quality is acceptable for video streaming applications. It can be noticed that the threshold $t$ is very delicate to define as it depends on the granularity expected. If $t$ is high, it will result in high quality because the scheme will grant all network capacity to a small number of flows. Nevertheless, this restriction raises underutilization problem, which is expensive for service providers. With the same reasoning, if $t$ is small, it will result in low quality due to congestion in the network. Thus, a tradeoff between bandwidth utilization (accepting more connections) and its consequence in connection degradation has to be well weighed.

\section{B. Interaction between Access Points and PSQA}

We assume all access points in our scheme have two more capabilities: monitoring loss and communicating with PSQA. The PSQA tool operates at every access point and helps them for MOS computation. The interaction between access point and PSQA tool is explained and illustrate as follow.

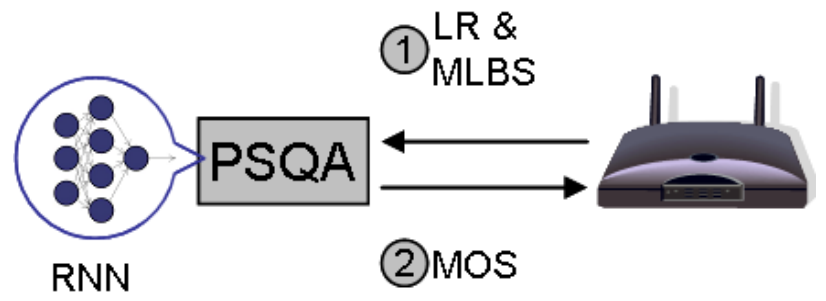

Fig. 3. Interaction between the access point and PSQA tool

1) The access point monitors loss statistics of each caring station and gives these statistics to PSQA tool as input. We focused on two specific parameters concerning losses because previous work from PSQA has demonstrated that the loss statistics is the most important factor for quality. Therefore, the statistics considered in our implementation are loss rate (LR) and mean loss burst size (MLBS). LR is the loss rate of video packets and MLBS is the average length of a sequence of consecutive lost packets, this parameter captures the way losses are distributed in the flow.

2) After receiving statistics from the access point, PSQA tool computes MOS and returns it to the access point.

\section{Exemplar Scenario}

In this section, we first illustrate the effect of no admission control in the system and then we explain how our scheme will be applied to this kind of situation.

Assuming in this example that the service provider does not implement any admission control mechanism; the connection arrival rate is one connection per second and the service 
provider accepts all connections until its maximum capacity. We run simulations and observe how quality evaluates in time. From the Fig.4, we can see that QoE is excellent at the beginning because a small number of connections can profit from all available bandwidth. However, when the number of admitted connections gets to 11 , the quality begins to degrade until it reaches and remains at the score 1 (bad quality).

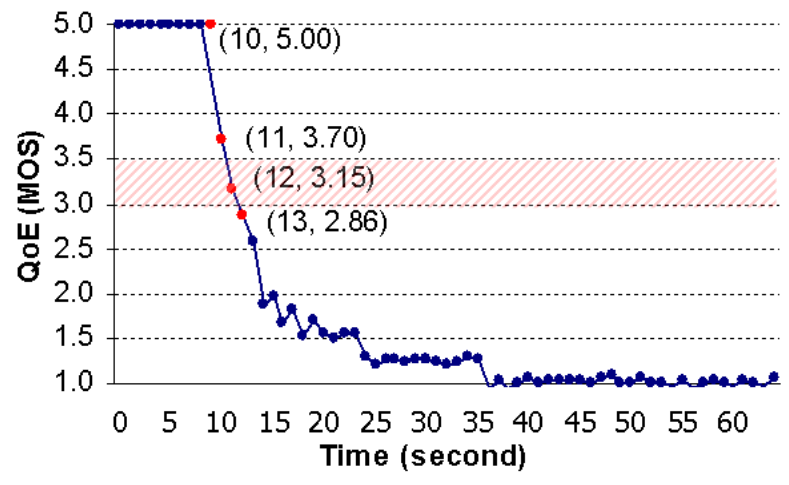

Fig. 4. QoE in the example scenario

We illustrate the functioning of our scheme by an example in Fig.4. In this example, we adopt a value of 0.5 for the threshold $t$ because after extensive simulations we found that the value chosen provides a good balance between bandwidth utilization and quality degradation. With $t=0.5$, the service provider will accept connections until current MOS reaches an interval [3.0-3.5] and then stop admitting new connection at 12 admitted flows where this threshold is attained (hatched zone). The number of flows remains 12 until at least one connection releases its bandwidth and the provider can then accept a new flow again.

It is noticed that the threshold $t$ has a great impact on the number of admitting connection. In the future, we could use different thresholds to treat different user priorities. For example, user with high priority will have a high threshold because the perceived quality have to be guaranteed strictly. On the other hands, the threshold for lower priority users may be smaller because this class of users is less sensitive or less restricted in terms of quality.

\section{IMPLEMENTATION}

We consider wireless access network based on IEEE 802.11 b specification [10] and we evaluate our scheme with video streaming application. For the simulation, we use the network simulator NS-2 [11] version 2.28 with the wireless update patch from TKN [12].

\section{A. System configuration}

The admission control is implemented in an access point operating in infrastructure mode. This access point is situated in the middle of the cell possessing a coverage area of $500 \mathrm{~m} \times 500 \mathrm{~m}$. Client nodes are positioned randomly in the cell. Each client requests for video streaming of $360 \mathrm{kbps}$ with connection handling time of 64 seconds. Connection arrival is one connection per second. The access point monitors MOS for each connection with an assumption that it is capable of computing MOS of each connection using statistics measured at its downlink interfaces.

In our system, we take an example of video streaming application in wireless networks as explained in the exemplar scenario. We have trained and validated a version of PSQA tool for this application. We take the threshold $t=0.5$ after extensive simulations and we believe that it is the appropriate value to protect overall quality.

\section{B. Comparison with loss rate based schemes}

We compare our proposed scheme with admission control implementation based on loss rate because it is the most pertinent metric to determine quality. We choose three ratebased schemes $(2 \%, 5 \%$, and $10 \%)$ corresponding to low, medium, and high loss for video streaming application in the wireless system. In each loss-based scheme, the access point will stop admitting new connection when the specified percentage of loss is reached. The result of evaluation is detailed in Section V.

\section{Performance Evaluation}

We consider two significant metrics to evaluate our scheme. The first one is user satisfaction that we measure in terms of MOS and the second one is goodput. We detailed our result according to these metrics and give the summary of performance comparison.

\section{A. User Satisfaction}

For measuring user satisfaction, we should evaluate how users perceive the service and how satisfied are they. To do so, we deploy PSQA tool to measure MOS of each connection, which can be translated into user perception of the service. We illustrate here the global MOS of the system by taking an average MOS of all active connections; this is done every decision epoch determined by the connection arrival rate. In our system, this is done every second, thus the MOS presented in Fig. 5 is taken every second. In addition, we also continue to measure MOS during transmission periodically to show how MOS evaluates in time.

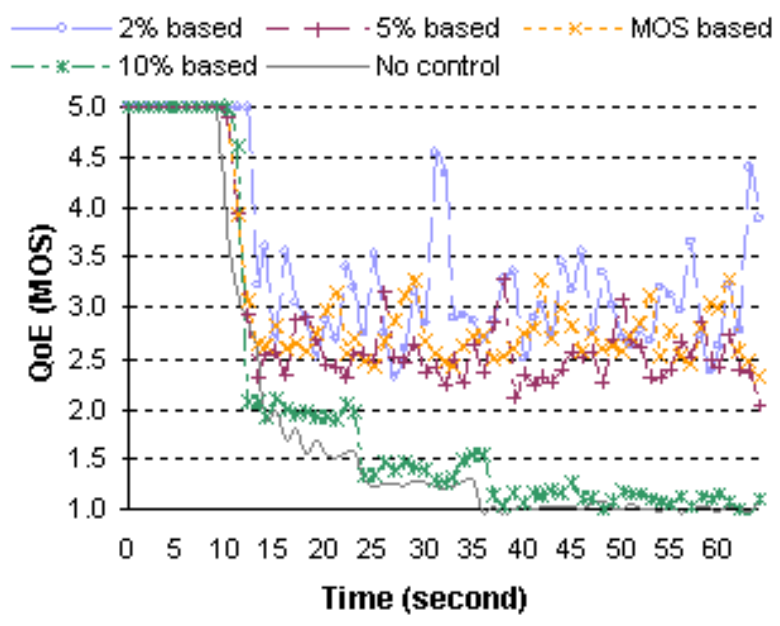

Fig. 5. QoE and loss based schemes: MOS comparison 
From the Fig.5, it can be seen that our scheme outperforms no-control approach and 10\% based approach. This can be explained by the fact that in the no-control approach, the call admission control does not exist and the network always accepts new connections leading to congestion and hence bad quality. In the second case, limiting loss rate at $10 \%$ is too high for obtaining good quality for video streaming applications. Our scheme performs slightly better than $5 \%$ based approach which is, generally, a delimited loss rate beyond which quality will no longer be acceptable. Nevertheless, $2 \%$ based approach gives better scores than ours does but with the price of bandwidth underutilization.

\section{B. Goodput}

Goodput is the application level throughput, which means the number of useful bits per unit of time forwarded by the network from a source to a destination. For measuring the goodput in NS-2, we measure the number of bits successfully received at each station. Fig. 6 shows the global goodput of the network in each scheme. The result confirms less goodput obtained by $2 \%$ based scheme as we just mentioned earlier. Indeed the $2 \%$ of loss is very restricted for admission, consequently the goodput of $2 \%$ based scheme is lower than others. We can also observe that $5 \%$ based scheme perform pretty well in the beginning but the throughput drops sharply around the 35th second. On the contrary, our scheme performs slightly lower at the beginning but it maintains at good level until around 50th second, while the others perform worst.

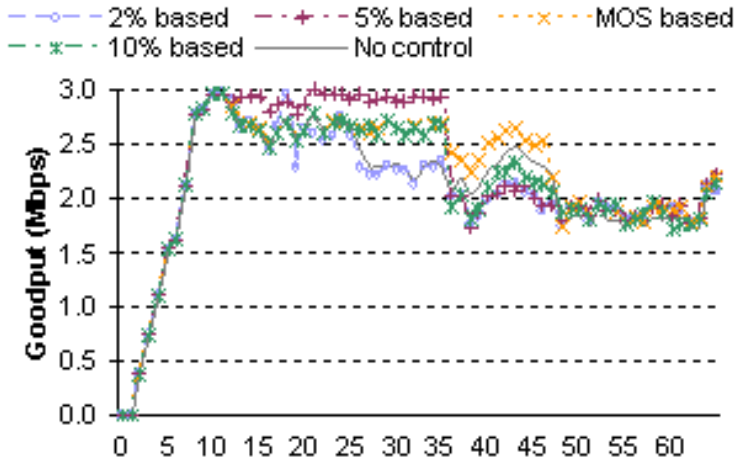

Time (second)

Fig. 6. QoE and loss based schemes: Goodput comparison

\section{Summary of performances}

TABLE II

COMPARING QOE AND LOSS BASED SCHEMES: SUMMARY

\begin{tabular}{|c||c|c|c|}
\hline Scheme & $\begin{array}{c}\text { Max. Bandwidth } \\
\text { utilization }\end{array}$ & $\begin{array}{c}\text { Connection } \\
\text { admitted }\end{array}$ & $\begin{array}{c}\text { Average } \\
\text { MOS }\end{array}$ \\
\hline \hline $2 \%$ based & $3.6 \mathrm{Mbps}$ & 10 flows & 3.62 \\
\hline MOS based & $\mathbf{4 . 3 2}$ Mbps & 12 flows & $\mathbf{3 . 3 5}$ \\
\hline $5 \%$ based & $3.96 \mathrm{Mbps}$ & 11 flows & 3.19 \\
\hline $10 \%$ based & $4.68 \mathrm{Mbps}$ & 13 flows & 2.17 \\
\hline No-control & $7.2 \mathrm{Mbps}$ & 20 flows & 2.06 \\
\hline
\end{tabular}

We summarize the performance of five schemes previously explained and highlight the performance of our mechanism in Table II. We also give information about number of flows admitted by each scheme and the maximum bandwidth utilization in the table.

\section{CONCLUSION}

In this paper, we have presented an admission control mechanism based on quality perception. We look at the resource management problem in a different angle. While others have considered technical parameters, we investigate the interpretation of these parameters for human that is QoE. Thus, the proposed scheme provides a method to dynamically control radio resource in wireless network while being aware of this QoE. Although the scheme only based on current score, we get a good performance. In the future, we will refine this scheme to enable MOS prediction and hence QoE provisioning. Moreover, As service differentiation is an important concern in wireless LANs nowadays, the threshold can be further used to address different user priorities.

\section{ACKNOWLEDGMENT}

This work has been done within the ANEMONE project, funded by the Sixth Framework Program of the European Commission's Information Society Technology.

\section{REFERENCES}

[1] "IEEE Std.802.11, Wireless LAN Medium Access Control (MAC) and Physical Layer (PHY) Specifications," New York, USA, 2007 (Revision of IEEE std 802.11-1999).

[2] "IEEE P802.11e/D13.0, Wireless LAN Medium Access Control (MAC) and Physical Layer (PHY) Specifications: Medium Access Control (MAC) Quality of Service (QoS) Enhancements," New York, USA, January 2005.

[3] S.-T. Sheu and T.-F. Sheu, "A bandwidth allocation/sharing/extension protocol for multimedia over IEEE 802.11 ad hoc wireless LANs," Selected Areas in Communications, IEEE Journal on, vol. 19, no. 10, pp. 2065-2080, Oct 2001.

[4] G. Bianchi, "Performance analysis of the IEEE 802.11 distributed coordination function," Selected Areas in Communications, IEEE Journal on, vol. 18, no. 3, pp. 535-547, March 2000.

[5] D. Pong and T. Moors, "Call admission control for IEEE 802.11 contention access mechanism," Global Telecommunications Conference, 2003. GLOBECOM '03. IEEE, vol. 1, pp. 174-178, 1-5 Dec. 2003.

[6] H. Zhai, X. Chen, and Y. Fang, "A call admission and rate control scheme for multimedia support over IEEE 802.11 wireless LANs," Wirel. Netw., vol. 12, no. 4, pp. 451-463, 2006.

[7] Y. Park, Y. Seok, N. Choi, Y. Choi, and J.-M. Bonnin, "Rate-adaptive multimedia multicasting over IEEE 802.11 wireless LANs," Consumer Communications and Networking Conference, 2006. CCNC 2006. 3rd IEEE, vol. 1, pp. 178-182, 8-10 Jan. 2006.

[8] "Definition of Quality of Experience (QoE)," Reference: TD 109rev2 (PLEN/12), ITU - International Telecommunication Union.

[9] G. Rubino, M. Varela, and J.-M. Bonnin, "Controlling Multimedia QoS in the Future Home Network Using the PSQA Metric," Computer Journal, vol. 49, no. 2, pp. 137-155, 2006.

[10] "IEEE Std.802.11, Wireless LAN Medium Access Control (MAC) and Physical Layer (PHY) Specifications: Higher-Speed Physical Layer Extension in the $2.4 \mathrm{GHz}$ Band," New York, USA, 1999 (R2003).

[11] "The Network Simulator - NS-2," http://www.isi.edu/nsnam/ns/.

[12] “TKN - Telecommunication and Networks Group," Technical University of Berlin, Germany, http://www.tkn.tu-berlin.de/. 